Pacific Journal of Mathematics

FREDHOLM THEORY OF PARTIAL DIFFERENTIAL
EQUATIONS ON COMPLETE RIEMANNIAN MANIFOLDS 


\title{
FREDHOLM THEORY OF PARTIAL DIFFERENTIAL EQUATIONS ON COMPLETE RIEMANNIAN MANIFOLDS
}

\author{
ROBERT C. MCOWEN
}

\begin{abstract}
This paper studies necessary and sufficient conditions for differential operators to be Fredholm on the Sobolev spaces of a complete (not necessarily compact) Riemannian manifold $\Omega$. The conditions are formulated algebraically in terms of the nonvanishing of the operator's principal symbol on $\Omega$ (ellipticity) and its "total symbol" at infinity of $\Omega$. The operators considered arise by taking sums of products of vector fields, all of whose covariant derivatives vanish at infinity; and the study involves $C^{*}$-algebra techniques. The required technical restrictions on the curvature and topology of $\Omega$ near infinity are much weaker than those in earlier joint work with $H$. O. Cordes.
\end{abstract}

o. Introduction. Let $\Omega$ be an $n$-dimensional paracompact $C^{\infty}$ manifold with complete Riemannian metric $d s^{2}=g_{i j} d x^{i} d x^{j}$ and surface measure $d \mu=\sqrt{g} d x$ where $g=\operatorname{det}\left(g_{i j}\right)$. Let $\Delta=g^{i j} \nabla_{i} \nabla_{j}$, the Beltrami-Laplace operator on $\Omega$, where $\left(g^{i j}\right)=\left(g_{i j}\right)^{-1}$ and $\nabla$ denotes covariant differentiation with respect to the Riemannian connection. Then $\Lambda=(1-\Delta)^{-1 / 2}$ is a positive-definite operator in $\mathscr{L}(\mathfrak{E})$, the bounded operators over the Hilbert space $\mathscr{S}=L^{2}(\Omega, d \mu)$. Define the $N$ th-Sobolev space $\mathfrak{S}_{N} \subset \mathfrak{F}$ for $N=1,2, \cdots$ by requiring $\Lambda^{N}: \mathfrak{F}_{\rightarrow} \rightarrow \mathfrak{F}_{N}$ to be an isometric isomorphism. It was shown in [3] that $C_{0}^{\infty}(\Omega)$ is dense in each $\mathfrak{S}_{N}$.

Now suppose we are given a differential operator $L$ on $\Omega$, of order $N$, such that we obtain a bounded map $L: \mathfrak{F}_{N} \rightarrow \mathfrak{F}_{2}$. We may ask the question when is $L$ Fredholm (i.e., when are ker $L$ and coker $L$ finite-dimensional subspaces of $\mathfrak{S}_{N}$ and $\mathfrak{S}$ respectively)? If $\Omega$ is compact, Seeley [13] showed that $L$ is Fredholm if and only if $L$ is elliptic (i.e., the principal symbol of $L$ never vanishes on the cosphere bundle $S^{*} \Omega$ ). For the case $\Omega=\boldsymbol{R}^{n}$, on the other hand, ellipticity is not sufficient to imply $L$ is Fredholm; Cordes and Herman [4] derived necessary and sufficient Fredholm criteria in terms of the "total symbol" of $L$.

In [4] the techniques involved considering operators $L \Lambda^{N}$ as generators of a $C^{*}$-algebra $\mathfrak{A} \subset \mathscr{L}(\mathfrak{S})$ which is commutative modulo the compact ideal, $\mathscr{K}$. The symbol of $L \Lambda^{N}$ is then defined as the continuous function $\sigma_{L \Lambda^{N}}$ on the maximal ideal space $\boldsymbol{M}$ of $\mathfrak{A} / \mathscr{K}$ provided by the Gel'fand theory. Thus $L \Lambda^{N}$ is Fredholm if and only 
if $\sigma_{L A^{N}}$ is never zero on $M$, and algebraic criteria are obtained by giving a precise description of $M$ and $\sigma_{L A^{N}}$.

In this paper we shall attempt to generalize the results of [4] and [13] to noncompact Riemannian manifolds $\Omega$. We shall use techniques similar to those in [4], generating a $C^{*}$-algebra $\mathfrak{A}$ which is commutative modulo $\mathscr{K}$, and then attempting to describe the maximal ideal space $M$ and functions $\sigma_{L \Lambda^{N}}$. Of course a precise description of $\boldsymbol{M}$ can only be made for specified $\Omega$; but our main result, Theorem 4.1, yields a sufficient condition for $L: \mathfrak{S}_{N} \rightarrow \mathfrak{S C}_{\text {to }}$ be Fredholm, namely that the "formal algebra symbol" $\dot{\sigma}_{L \Lambda} N$ never vanish on the space $\partial P^{*} \Omega$ (see $\S 4$ for definitions). Thus operators such as $(\lambda-\Delta)^{N}$ are Fredholm on the Sobolev spaces of $\Omega$ for $\lambda>0$, whereas for $\lambda=0$ we need to use weighted Sobolev spaces (see [2] and [11]). On the other hand, ellipticity is certainly a necessary condition for $L$ to be Fredholm (c.f. §2).

This paper summarizes the principal results of the author's Ph.D. thesis, and generalizes earlier joint work with his advisor, H. O. Cordes, whose contributions to this research are also gratefully acknowledged.

1. The $C^{*}$-algebra for singular elliptic theory. We begin by describing the (global) differential operators that we will be considering. Suppose $l_{\alpha}$ is a $C^{\infty}$-contravariant tensor field of degree $\alpha \geqq 0$ on $\Omega$. Then in local coordinates $l_{\alpha}$ has components $l_{\alpha}^{i_{1} \cdots i_{\alpha}}$, and we may define a differential operator $l_{\alpha} \nabla^{\alpha}$ by

$$
l_{\alpha} \nabla^{\alpha} u=l_{\alpha}^{i_{1} \cdots i_{\alpha}} \nabla_{i_{1}} \cdots \nabla_{i_{\alpha}} u
$$

for $u \in C_{0}^{\infty}(\Omega)$. (Note here and throughout this paper we employ the usual summation convention for tensor indices.) The differential operators we consider are just sums $L=\sum_{\alpha=0}^{N} l_{\alpha} \nabla^{\alpha}$.

For any tensor field $T$, we shall denote its length (a function over $\Omega$ ) by $|T|$. In order to analyze the behavior of our operators "at infinity" let us make the following definition: if $f$ is a (complexvalued) function on $\Omega$, we shall write $\lim _{x \rightarrow \infty} f=M$ provided for any $\varepsilon>0$ there is a compact set $K \subset \Omega$ such that $|f(x)-M|<\varepsilon$ for all $x \in \Omega \backslash K$. We shall consider the following conditions on the curvature tensor $R$.

Condition $H . \quad\left|\nabla^{k} R\right|$ is bounded over $\Omega$ for all $k \geqq 0$.

Condition $H_{0}$. In addition to Condition 'H, $\lim _{x \rightarrow \infty}\left|\nabla^{k} R\right|=0$ for all $k \geqq 1$.

Throughout this paper we shall be assuming Condition $\mathrm{H}$ with- 
out further mentioning it.

We may now prove the following

THEOREM 1.1. If $L=\sum_{\alpha=0}^{N} l_{\alpha} \nabla^{\alpha}$ has bounded coefficients (i.e., each $\left|l_{\alpha}\right|$ is bounded over $\left.\Omega\right)$, then $L \Lambda^{N} \in \mathscr{L}(\mathfrak{S})$. If in addition $L$ has coefficients vanishing at infinity (i.e., $\lim _{x \rightarrow \infty}\left|l_{\alpha}\right|=0$ for each $\alpha$ ), then $\Lambda L \Lambda^{N} \in \mathscr{K}(\mathfrak{E})$.

Proof. It suffices to consider top-order terms only: $L=l_{N} \nabla^{N}$. Let $\psi \in C_{0}^{\infty}(\Omega)$. Condition $\mathrm{H}$ implies the following estimate for the $L^{2}$-norms:

$$
\left\|\nabla^{N} \psi\right\|^{2} \leqq C\left(\sum_{0 \leqq k \leqq(N / 2)}\left\|\Delta^{k} \psi\right\|^{2}+\sum_{0 \leqq k \leqq(N-1 / 2)}\left\|\nabla \Delta^{k} \psi\right\|^{2}\right)
$$

where $C$ is a constant. (Note: if $T$ is a tensor with length $|T|$, then $\|T\|^{2}=\int_{\Omega}|T|^{2} d \mu$. C. f. Aubin [1] for this notation, and Proposition 3 in [1] for the estimate (1.2).) So

$$
\begin{aligned}
\|L \psi\|^{2} & \leqq\left(\sup _{x \in \Omega}\left|l_{N}\right|^{2}\right)\left\|\nabla^{N} \psi\right\|^{2} \\
& \leqq C_{1}\left(\sum_{0 \leqq k \leqq(N / 2)}\left\|\Delta^{k} \psi\right\|^{2}+\sum_{0 \leqq k \leqq(N-1 / 2)}\left\|\nabla \Delta^{k} \psi\right\|^{2}\right) \\
& \leqq C_{2}\left(\sum_{0 \leqq k \leqq(N / 2)}\left\|(1-\Delta)^{k} \psi\right\|^{2}+\sum_{0 \leqq k \leqq(N-1 / 2)}\left\|\nabla(1-\Delta)^{k} \psi\right\|^{2}\right) \\
& \leqq C_{3}\left\|(1-\Delta)^{N / 2} \psi\right\|^{2}
\end{aligned}
$$

where the $C_{i}$ are constants and for the last inequality we have used the estimates (for $\phi \in C_{0}^{\infty}(\Omega)$ )

$$
\begin{aligned}
& \|\phi\|^{2} \leqq\left\|(1-\Delta)^{1 / 2} \phi\right\|^{2} \leqq\|(1-\Delta) \phi\|^{2} \\
& \|\nabla \phi\|^{2}=\left\|(1-\Delta)^{1 / 2} \phi\right\|^{2}-\|\phi\|^{2} \leqq\left\|(1-\Delta)^{1 / 2} \phi\right\|^{2} .
\end{aligned}
$$

Since $\Lambda^{-N} C_{0}^{\infty}(\Omega)$ is dense in $\mathscr{S}$ (c.f. Cordes [3]), estimate (1.3) proves $L \Lambda^{N} \in \mathscr{L}(\mathfrak{S})$. Now suppose $\lim _{x \rightarrow \infty}\left|l_{N}\right|=0$ and for $\varepsilon>0$ choose $K \subset \Omega$, compact, such that $\left|l_{N}\right|<\varepsilon$ for $x \in \Omega \backslash K$. Let $\phi \in C_{0}^{\infty}(\Omega)$ with supp $\phi \subset K$ and define $L_{\varepsilon}=\phi L$. Then $\Lambda L_{\varepsilon} \Lambda^{N}=\Lambda \phi \cdot L \Lambda^{N} \in \mathscr{K}(\mathfrak{K})$, since $L \Lambda^{N} \in \mathscr{L}(\mathfrak{S})$ and $\Lambda \phi \in \mathscr{K}(\mathscr{S})$ by Theorem 3.1 in [5]. Since $\Lambda L_{\varepsilon} \Lambda^{N} \rightarrow$ $\Lambda L \Lambda^{N}$ in norm as $\varepsilon \rightarrow 0$, we also have $\Lambda L \Lambda^{N} \in \mathscr{K}(\mathfrak{S})$.

In order to do Fredholm theory, we must restrict our attention to the following classes of operators 


$$
\begin{gathered}
\boldsymbol{T}^{N}=\left\{L=\sum_{\alpha=0}^{N} l_{\alpha} \nabla^{\alpha}: \text { each }\left|l_{\alpha}\right| \text { is bounded over } \Omega\right. \text { and satisfies } \\
\left.\lim _{x \rightarrow \infty}\left|\nabla^{k} l_{\alpha}\right|=0 \text { for all } k \geqq 1\right\} .
\end{gathered}
$$

Let $\boldsymbol{T}_{0}^{N} \subset \boldsymbol{T}^{N}$ be those operators for which $\lim _{x \rightarrow \infty}\left|l_{\alpha}\right|=0$ for each $\alpha$. We shall denote the function algebra $\boldsymbol{T}^{0}$ by $\boldsymbol{A}$, and the collection of vector fields $L=l_{1} \nabla^{1} \in \boldsymbol{T}^{1}$ by $\boldsymbol{D}$. Let $\boldsymbol{L}^{N}$ be the subcollection of $\boldsymbol{T}^{N}$ generated by taking sums of products of functions in $\boldsymbol{A}$ and vector fields in $\boldsymbol{D}$. Finally, let $\boldsymbol{A}_{0}=\boldsymbol{T}_{0}^{0}, \boldsymbol{D}_{0}=\boldsymbol{D} \cap \boldsymbol{T}_{0}^{1}$, and $\boldsymbol{L}_{0}^{N}=\boldsymbol{L}^{N} \cap \boldsymbol{T}_{0}^{N}$. Note that the $\boldsymbol{T}^{N}$ and $\boldsymbol{L}^{N}$ form graded algebras with respect to ordinary operator product, and that $\boldsymbol{D}$ is a Lie algebra under commutator product with $D_{0}$ as an ideal. Also note that all classes defined are $\boldsymbol{A}$-modules.

For $L=\sum_{\alpha=0}^{N} l_{\alpha} \nabla^{\alpha} \in \boldsymbol{T}^{N}$, we define the formal adjoint by $L^{\prime}=$ $\sum_{\alpha=0}^{N}(-1)^{\alpha} \nabla^{\alpha} \bar{l}_{\alpha}$ where the operator $\nabla^{\alpha} \bar{l}_{\alpha}$ is defined analogously to (1.1):

$$
\left.\nabla^{\alpha} \bar{l}_{\alpha} u=\nabla_{i_{1}} \cdots \nabla_{i_{\alpha}} \overline{\left(l_{\alpha}^{i_{1} \cdots i_{\alpha}}\right.} u\right)
$$

for $u \in C_{0}^{\infty}(\Omega)$. Using the Leibniz rule for covariant derivatives, note that $L^{\prime}=(-1)^{N} \bar{L}+$ lower order terms $\in \boldsymbol{T}^{N}$. Also note that $L \in$ $\boldsymbol{L}^{N}\left(\right.$ resp. $\left.\boldsymbol{T}_{0}^{N}\right)$ implies $L^{\prime} \in \boldsymbol{L}^{N}\left(\operatorname{resp} . \boldsymbol{T}_{0}^{N}\right)$. The formal adjoint has the property that for $u, v \in C_{0}^{\infty}(\Omega)$ we have $\langle L u, v\rangle=\left\langle u, L^{\prime} v\right\rangle$ (where $\langle$,$\rangle denotes the L^{2}$ inner product). Thus, using self-adjointness of $\Lambda$, we have $\left\langle L \Lambda^{N} u, v\right\rangle=\left\langle u, \Lambda^{N} L^{\prime} v\right\rangle$ for $v \in C_{0}^{\infty}(\Omega)$ and $u \in \Lambda^{-N} C_{0}^{\infty}(\Omega)$. Since both $C_{0}^{\infty}(\Omega)$ and $\Lambda^{-N} C_{0}^{\infty}(\Omega)$ are dense in $\mathfrak{S}$ we obtain the following from Theorem 1.1.

CoRollary 1.2. If $L \in T^{N}$, then $L \Lambda^{N}, \Lambda^{N} L \in \mathscr{L}(\mathfrak{S})$ and $\left(L \Lambda^{N}\right)^{*}=$ $\Lambda^{N} L^{\prime}$ (where * denotes the Hilbert space adjoint).

We next begin investigating commutators. Let $\boldsymbol{L}^{N}+\boldsymbol{T}_{0}^{N}$ denote the algebraic sum of those two classes of operators.

LemmA 1.3. If $L \in \boldsymbol{L}^{N_{1}}+\boldsymbol{T}_{0}^{N_{1}}$ and $M \in \boldsymbol{L}^{N_{2}}+\boldsymbol{T}_{0}^{N_{2}}$ then $[L, M] \epsilon$ $\boldsymbol{T}_{0}^{N_{1}+N_{2}-1}$ and $[L, \Delta] \in \boldsymbol{T}_{0}^{N_{1}+1}$.

Proof. If $L \in \boldsymbol{T}_{0}^{N_{1}}$ or $M \in \boldsymbol{T}_{0}^{N_{2}}$, then $[L, M] \in \boldsymbol{T}_{0}^{N_{1}+N_{2}-1}$ may easily be seen by collecting top-order terms. Hence we may assume $L \in \boldsymbol{L}^{N_{1}}$ and $M \in \boldsymbol{L}^{N_{2}}$. The proof of the first statement then follows using $\left[D_{1}, D_{2}\right] \in D_{0}$ and $[L, D M]=[L, D] M+D[L, M]$ for $D, D_{1}, D_{2} \in D$. To prove the second statement we may assume $L \in L^{N_{1}}$, and has top terms only: $L=D_{1} \cdots D_{N_{1}}$ where $D_{j} \in D$. Then $[L, \Delta]=$ $\left[D_{1} \cdots D_{N_{1}}, \Delta\right]=D_{1} \cdots D_{N_{1}-1}\left[D_{N_{1}}, \Delta\right]+\left[D_{1} \cdots D_{N_{1}-1}, \Delta\right] D_{N_{1}}$ so the second 
statement follows provided we can show $[D, \Delta] \in \boldsymbol{T}_{0}^{2}$ for every $D \in \boldsymbol{D}$. But letting $D=b^{i} V_{i}$, a computation in local coordinates shows

$$
\begin{aligned}
{[D, \Delta] } & =\left[b^{i} \nabla_{i}, g^{j k} \nabla_{j} \nabla_{k}\right] \\
& \equiv b^{i} g^{j k} \nabla_{i} \nabla_{j} \nabla_{k}-b^{i} g^{j k} \nabla_{j} \nabla_{k} \nabla_{i}\left(\bmod \boldsymbol{T}_{0}^{2}\right) \\
& =b^{i} g^{j k} R_{k j i}^{l} \nabla_{l} \\
& =b^{i} g^{l p} R_{i p k}^{k} \nabla_{l} \\
& =-g^{l p}\left(\nabla_{k} \nabla_{p} b^{k}-\nabla_{p} \nabla_{k} b^{k}\right) \nabla_{l} \\
& \equiv 0\left(\bmod \boldsymbol{T}_{0}^{2}\right) .
\end{aligned}
$$

This lemma and Theorem 1.1 enable us to investigate commutators of certain bounded operators.

LEMma 1.4. If $L \in \boldsymbol{L}^{N}+\boldsymbol{T}_{0}^{N}$, then $\left[L \Lambda^{N}, \Lambda\right]=K_{1} \Lambda=\Lambda K_{2}$ where $K_{j} \in \mathscr{K}(\mathfrak{E})$.

Proof. If we let $R(\lambda)=\left(\lambda-\Lambda^{2}\right)^{-1}$ and $\Gamma=\{z \in C:|z-1|=1\}$ (with positive orientation), then

$$
\left[L \Lambda^{N}, \Lambda\right]=\frac{1}{2 \pi i} \int_{\Gamma} \sqrt{\lambda}\left[L \Lambda^{N}, R(\lambda)\right] d \lambda
$$

and

$$
\begin{aligned}
{\left[L \Lambda^{N}, R(\lambda)\right] } & =R(\lambda)\left[L \Lambda^{N-1}, \Lambda^{2}\right] R(\lambda) \Lambda \\
& =R(\lambda) \Lambda^{2}[L, \Delta] \Lambda^{N+1} R(\lambda) \Lambda \\
& =\Lambda R(\lambda) K R(\lambda) \Lambda
\end{aligned}
$$

where $K=\Lambda[L, \Delta] \Lambda^{N+1} \in \mathscr{K}$ by Theorem 1.1 and Lemma 1.3. Let $K_{1}(\lambda)=\Lambda R(\lambda) K R(\lambda)$ and $K_{2}(\lambda)=R(\lambda) K R(\lambda) \Lambda$, so $K_{j}(\lambda)$ defines a norm continuous map from $\Gamma \backslash\{0\}$ to $\mathscr{K}$. Using (4.1) of [5] with $s=1$ shows that $K_{j}(\lambda)=O\left(|\lambda|^{-3 / 4}\right)$. So $K_{j}=(2 \pi i)^{-1} \int_{\Gamma} \sqrt{\lambda} K_{j}(\lambda) d \lambda \in \mathscr{K}$ for $j=1,2$ and the lemma is proved.

The above lemma enables us to prove the following useful result.

Proposition 1.5. If $L \in L^{N}+T_{0}^{N}$ and $P$ and $Q$ are nonnegative integers such that $P+Q=N$, then

(i) $\Lambda^{P} L \Lambda^{Q} \in \mathscr{L}(\mathfrak{F})$ and (ii) $\Lambda^{P} L \Lambda^{Q}-L \Lambda^{N} \in \mathscr{K}^{r}(\mathfrak{E})$. In addition, if $L \in T_{0}^{N}$ then (iii) $\Lambda^{P+1} L \Lambda^{Q} \in \mathscr{K}(\mathfrak{S})$.

Proof. We perform an induction on $P$. The case $P=0$ is just Theorem 1.1: for $N \geqq 0$ and $L \in L^{N}+T_{0}^{N}$ we have (i) $L \Lambda^{N} \in \mathscr{L}(\mathfrak{S})$, (ii) $L \Lambda^{N}-L \Lambda^{N} \in \mathscr{K}$, and (in case $L \in T_{0}^{N}$ ) (iii) $\Lambda L \Lambda^{N} \in \mathscr{K}$. Now 
assume by induction that the proposition is true for $P$. For $N \geqq P$, let $L \in L^{N}+T_{0}^{N}$ and $Q=N-P$. Then

$$
\left[\Lambda^{P} L \Lambda^{Q}, \Lambda\right]=(2 \pi i)^{-1} \int_{\Gamma} \sqrt{\lambda}\left[\Lambda^{P} L \Lambda^{Q}, R(\lambda)\right] d \lambda
$$

and

$$
\begin{aligned}
{\left[\Lambda^{P} L \Lambda^{Q}, R(\lambda)\right] } & =R(\lambda) \Lambda^{P}\left[L \Lambda^{Q-1}, \Lambda^{2}\right] R(\lambda) \\
& =\Lambda R(\lambda) \Lambda^{P+1}[L, \Delta] \Lambda^{Q+1} R(\lambda) \\
& =\Lambda R(\lambda) K R(\lambda) \Lambda
\end{aligned}
$$

where $\Gamma$ and $R(\lambda)$ are as in the preceding proof and $K=\Lambda^{P+1}[L, \Delta] \Lambda^{2+1}$. But by Lemma $1.3,[L, \Delta] \in \boldsymbol{T}_{0}^{N+1}$, so $K=\Lambda\left(\Lambda^{P}[L, \Delta] \Lambda^{Q+1}\right) \in \mathscr{K}$ by the induction hypothesis (for $P$ and $N+1$ ). So if we let $K_{1}(\lambda)=$ $A R(\lambda) K R(\lambda)$ and $K_{2}=R(\lambda) K R(\lambda) \Lambda$, then $K_{j}(\lambda)=O\left(|\lambda|^{-3 / 4}\right)$ and $\left[\Lambda^{P} L \Lambda^{Q}, \Lambda\right]=K_{1} \Lambda=\Lambda K_{2}$ where $K_{j}=(2 \pi i)^{-1} \int_{\Gamma} K_{j}(\lambda) d \lambda \in \mathscr{K}$ for $j=$ 1, 2. Since $\mathfrak{K}_{1}=\Lambda \mathfrak{K}$ is dense in $\mathfrak{E}$, this implies

$$
\Lambda^{P} L \Lambda^{Q}-\Lambda^{P+1} L \Lambda^{Q-1} \in \mathscr{K} \text {. }
$$

Using the induction hypothesis, we get (i) for $P+1$. In fact the induction hypothesis also yields

$$
\Lambda^{P} L \Lambda^{Q}-L \Lambda^{N} \in \mathscr{K}
$$

which together with (1.11) yields (ii) for $P+1$. Finally, if $L \in \boldsymbol{T}_{0}^{N}$, multiplying (1.11) on the left by $\Lambda$ and invoking the case $P$ once more yields (iii) for $P+1$.

\section{CoRollary 1.6. If $L \in \boldsymbol{L}^{N}+\boldsymbol{T}_{0}^{N}$, then $L \Lambda^{N}-\Lambda^{N} L \in \mathscr{K}$.}

Let $\mathfrak{U}^{0}$ denote the algebra of bounded operators on $\mathfrak{S}$ which is finitely generated by $L \Lambda^{N}$ and $\Lambda^{N} L$ for $L \in L^{N}+T_{0}^{N}$. Note that $\mathfrak{A}^{0}$ contains $\Lambda$ and multiplications by functions in $\boldsymbol{A}$. Let $\mathfrak{A}$ be the norm closure of the collection $\mathfrak{X}^{0}+\mathscr{K}$. We may now prove the following.

THEOREM 1.7. 2 is a $C^{*}$-subalgebra of $\mathscr{L}(\mathfrak{S})$ with compact commutators.

Proof. $\quad \mathfrak{A}$ is closed under adjoints by Corollary 1.2. Concerning commutators, let $L_{1} \in \boldsymbol{L}^{N_{1}}+\boldsymbol{T}_{0}^{N_{1}}$ and $L_{2} \in \boldsymbol{L}^{N_{2}}+\boldsymbol{T}_{0}^{N_{2}}$ and suppose $N_{1} \geqq N_{2}$. Then $L_{2} \in \boldsymbol{L}^{N_{1}}+\boldsymbol{T}_{0}^{N_{1}}$ and

$$
\begin{aligned}
L_{1} \Lambda^{N_{1}} L_{2} \Lambda^{N_{2}} & \equiv L_{1} L_{2} \Lambda^{N_{1}} \Lambda^{N_{2}} \equiv \Lambda^{N_{1}} \Lambda^{N_{2}} L_{1} L_{2} \equiv \Lambda^{N_{2}} \Lambda^{N_{1}} L_{2} L_{1} \\
& \equiv \Lambda^{N_{2}} L_{2} \Lambda^{N_{1}} L_{1} \equiv L_{2} \Lambda^{N_{2}} L_{1} \Lambda^{N_{1}}(\bmod \mathscr{K})
\end{aligned}
$$


using Lemma 1.3 and Corollary 1.6. By Corollary 1.6 this takes care of all commutators.

We shall call $\mathfrak{A}$ the $C^{*}$-algebra for singular elliptic theory on $\Omega$. The reason for this is as follows. By Theorem 1.7, $2 / / \mathscr{K}$ is a commutative $C^{*}$-algebra; hence by the Gel'fand theory it is isometrically *-isomorphic to $C(\boldsymbol{M})$, the algebra of continuous functions on the space $\boldsymbol{M}$ of maximal ideals in $\mathfrak{U} / \mathscr{K}$. For $A \in \mathfrak{A}$, denote the image of its coset $A+\mathscr{K}$ in $C(\boldsymbol{M})$ by $\sigma_{A}$. Since $\mathfrak{A} / \mathscr{C}$ is a $C^{*}$ algebra, invertibility of $A+\mathscr{K}$ in $\mathscr{L}(\mathscr{K}) / \mathscr{C}$ is equivalent to its invertibility in $\mathfrak{X} / \mathscr{K}$, and hence to the nonvanishing of $\sigma_{A}$ on $\boldsymbol{M}$. Thus a further analysis of $\sigma_{A}$ and $\boldsymbol{M}$ is desired to provide an algebraic criterion for $A$ to be Fredholm. We turn to this question in the remaining sections.

2. Maximal ideals over finite points. Let $\mathfrak{A}_{m} \subset \mathfrak{A}$ denote the algebra of multiplication operators obtained by closing $\boldsymbol{A}$ under the uniform norm. Since $\mathfrak{A}_{m}$ is a closed *-subalgebra of all bounded continuous functions on $\Omega, \mathfrak{A}_{m}$ is isometrically *-isomorphic to $C(\bar{\Omega})$ where $\bar{\Omega}$ is some compactification of $\Omega$. Although $\bar{\Omega}$ has no simple geometrical structure, there is a continuous surjection $p: M \rightarrow \bar{\Omega}$ defined as the associated dual map (c.f. [12]) for the continuous injection $\mathfrak{A}_{m} \rightarrow \mathfrak{A} / \mathscr{C}$. Thus $\boldsymbol{S}=p^{-1}(\Omega)$ is the set of maximal ideals over finite points of $\Omega$. We can completely describe this part of $\boldsymbol{M}$ and $\sigma_{A}$. If $l_{\alpha}$ is a contravariant tensor field of degree $\alpha$, we shall denote by $l_{\alpha} \xi^{\alpha}$ the function defined on cotangent vectors $(x, \xi)$ by $l_{\alpha}^{i_{1} \cdots i_{\alpha}}(x) \xi_{i_{1}} \cdots \xi_{i_{\alpha}}$.

THEOREM 2.1. $\boldsymbol{S}$ is homeomorphic to $S * \Omega$, the bundle of unit cospheres, under the map $m \rightarrow(x, \xi)$ such that

$$
\sigma_{L 1^{N}}(m)=l_{N}(x) \xi^{N}
$$

where $L=\sum_{\alpha=0}^{N}(-i)^{\alpha} l_{\alpha} \nabla^{\alpha} \boldsymbol{L}^{N}+\boldsymbol{T}_{0}^{N}$. Thus uniform ellipticity is a necessary condition for $L: \mathfrak{S}_{N} \rightarrow \mathfrak{K}_{\mathrm{C}}$ to be Fredholm. (Note that as special cases of (2.1) we have $\sigma(m)=0$, and $\sigma_{a}(m)=a(x)$ for all $a \in \boldsymbol{A}$.)

Proof. For $m \in S$ with $p(m)=x \in \Omega$, let $x^{1}, \cdots, x^{n}$ be local coordinates in a chart $U$ which diagonalizes the metric at $x$. Let $N$ be a compact neighborhood of $x$ such that $N \subset U$, and let $\phi \in C_{0}^{\infty}(U)$ satisfying $0 \leqq \phi \leqq 1$ on $U$ and $\phi=1$ on $N$. Note that for each $\phi$, $S_{\nu}=-i \phi\left(\partial / \partial x^{\nu}\right) \Lambda \in \mathfrak{A}$ and $S_{\nu}^{*} \equiv S_{\nu}(\bmod \mathscr{\mathscr { C }})$. Hence $\xi_{\nu}=\sigma_{S_{\nu}}(m)$ is real for each $\nu$ and $(x, \xi)$ where $\xi=\sum_{\nu=1}^{n} \xi \nu d x^{\nu}$ defines an element of the cotangent space at $x$. Furthermore 


$$
\begin{aligned}
\sum_{\nu \mu} S_{\nu}^{*} g^{\nu \mu} S_{\mu} & =\sum_{\nu, \mu} \Lambda\left(\partial / \partial x^{\nu}\right) \phi g^{\nu \mu} \phi\left(\partial / \partial x^{\mu}\right) \Lambda \\
& \equiv-\Lambda \phi^{2} \Delta \Lambda(\bmod \mathscr{K}) \\
& \equiv-\phi^{2} \Lambda \Delta \Lambda(\bmod \mathscr{K}) \\
& \equiv \phi^{2}(\bmod \mathscr{K})
\end{aligned}
$$

So

$$
\begin{gathered}
|\xi|^{2}=\sum \xi_{\nu}^{2}=\sum_{\nu, \mu} \sigma_{S_{\nu}}(m) g^{\nu \mu}(x) \sigma_{S_{\mu}}(m) \\
=\sigma_{\left(\Sigma_{\nu, \mu} S_{\nu}{ }^{*} g^{\nu} \mu_{S_{\mu}}\right.}(m) \\
=\phi^{2}(x)=1 .
\end{gathered}
$$

Property (i) is immediate from the definition of the surjection $p: M \rightarrow \bar{\Omega}$ while (ii) follows from $\sigma_{\Lambda}(m)=\sigma_{\phi \Lambda}(m)=0$ since $\phi(x)=1$ and $\phi \Lambda \in \mathscr{K}$. The formula (2.1) holds for $N=1$ and $L=D=$ $-i b^{\nu}\left(\partial / \partial x^{\nu}\right) \in \boldsymbol{D}$ since

$$
\begin{aligned}
\sigma_{D \Lambda}(m) & =\sigma_{\phi^{2} D \Lambda}(m) \\
& =\sigma_{\left\{\Sigma_{\nu=1}^{n}\left(\phi b^{\nu}\right)\left(-i \phi \partial / \partial x^{\nu}\right) \Lambda\right\}}(m) \\
& =\sum_{\nu=1}^{n} b^{\nu}(x) \xi_{\nu}
\end{aligned}
$$

and this may be extended to higher-order tensors: for example $L=-l^{\nu \mu} \nabla_{\nu} \nabla_{\mu} \in \boldsymbol{L}^{2}+\boldsymbol{T}_{0}^{2}$ implies

$$
\phi^{2} L \Lambda^{2} \equiv \sum_{\nu, \mu}\left(-\phi l^{\nu \mu} \nabla_{\nu}\right)\left(\phi \nabla_{\mu}\right) \Lambda^{2} \equiv \sum_{\nu, \mu}\left(-\phi l^{\nu \mu} \nabla_{\nu} \Lambda\right)\left(\phi \nabla_{\mu} \Lambda\right)(\bmod \mathscr{K})
$$

so

$$
\begin{aligned}
\sigma_{L \Lambda^{2}}(m) & =\sigma_{\phi^{2} L \Lambda^{2}}(m) \\
& =\sum_{\nu, \mu}\left(-i l^{\nu \mu}(x) \xi_{\nu}\right)\left(i \xi_{\mu}\right) \\
& =l^{\nu \mu}(x) \xi_{\nu} \xi_{\mu} .
\end{aligned}
$$

The map $m \rightarrow(x, \xi)$ is clearly $1-1$. To show it is surjective onto $S^{*} \Omega$, we use the following lemma (whose proof is immediate) to achieve local rotations of $\boldsymbol{M}$.

LEMMA 2.2. Let $\phi \in \mathfrak{A}_{m}$ be real-valued satisfying $0 \leqq \phi \leqq 1$ on $\Omega$ and $\phi=1$ on a closed set $U \subset \bar{\Omega}$, and consider the $C^{*}$-algebra $\mathfrak{A}_{\phi} \subset \mathfrak{A}$ generated by $\mathscr{K}, a \in A, \phi \Lambda$, and $\phi^{N} L \Lambda^{N}: L \in L^{N}+T_{0}^{N}$. Let $M_{\phi}$ be the maximal ideal space of $\mathfrak{A}_{\phi} / \mathscr{K}, \sigma^{\phi}: \mathfrak{A}_{\phi} \rightarrow C\left(\boldsymbol{M}_{\phi}\right)$ the symbol homomorphism, $p_{\phi}: \boldsymbol{M}_{\phi} \rightarrow \bar{\Omega}$ the associated dual map to the inclusion $\mathfrak{A}_{m} \subset \mathfrak{A}_{\phi} / \mathscr{K}$. Let $\boldsymbol{N}_{\phi}=p_{\phi}^{-1}(U) \subset M_{\dot{\phi}}$ whereas $\boldsymbol{N}=p^{-1}(U) \subset \boldsymbol{M}$. Defining $\rho(\alpha+\mathscr{K})=$ $a+\mathscr{K}$ and $\rho\left(L \Lambda^{N}+\mathscr{K}\right)=\phi^{N} L \Lambda^{N}+\mathscr{K}$ yields a surjective algebra *-homomorphism $\rho: \mathfrak{A} / \mathscr{K} \rightarrow \mathfrak{A}_{\phi} / \mathscr{K}$ whose associated dual map 
provides an injection $j: M_{\phi} \rightarrow M$ such that

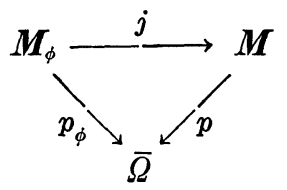

commutes and

$$
\sigma_{A}(j(n))=\sigma_{B}^{\phi}(n)
$$

for all $n \in M_{\phi}, A \in \mathfrak{A}$, and $B \in \rho(A+\mathscr{K})$. Furthermore, the restriction of $j$ to $N_{\phi}$ provides a homeomorphism onto $N$, so we may consider $\boldsymbol{N}_{\phi} \subset \boldsymbol{M}$ and $\boldsymbol{N} \subset \boldsymbol{M}_{\phi}$.

Applying the lemma in our case, since $\phi$ has compact support in a coordinate chart, the algebra $\mathfrak{A}_{\phi}$ is generated by $a \in A, S_{\nu}: \nu=$ $1, \cdots, n$, and $\mathscr{K}$. Choose any $\xi^{\prime} \in S_{x}^{*} \Omega$ and let $R=\left(r^{\nu \mu}\right)$ be an orthogonal matrix such that $\xi^{\prime}=R \xi$. Defining $\tau(a+\mathscr{K})=a+\mathscr{K}$ and $\tau\left(S_{\nu}+\mathscr{K}\right)=T_{\nu}+\mathscr{K}$ where $T_{\nu}=-i \phi \sum_{\mu=1}^{n} \gamma^{\nu \mu}\left(\partial / \partial x^{\mu}\right) \Lambda$, we obtain a surjective ${ }^{*}$-isomorphism $\tau: \mathfrak{A}_{\phi} / \mathscr{K} \rightarrow \mathfrak{A}_{\phi} / \mathscr{K}$ whose associated dual map must be a (surjective) homeomorphism $h: \boldsymbol{M}_{\phi} \rightarrow \boldsymbol{M}_{\phi}$ satisfying $\sigma_{A}^{\phi}(h(n))=\sigma_{B}^{\phi}(n)$ for all $A \in \mathfrak{A}_{\phi}, n \in \boldsymbol{M}_{\phi}$, and $B \in \tau(A+\mathscr{C})$. In particular, $h$ preserves fibers over $U$. Thus $m^{\prime}=j(h(m)) \in M$ has the desired property that $m^{\prime} \rightarrow(x, \xi)$ under the correspondence of the theorem since $\xi_{\nu}^{\prime}=\sum_{\mu=1}^{n} r^{\nu \mu} \xi_{\mu}=\sigma_{S_{\nu}}^{\phi}(h(m))=\sigma_{S_{\nu}}(j(h(m)))$. So we need only check continuity. But the topology of $M$ is defined so that each $\sigma_{A}$ is continuous, and since the functions (2.1) are continuous on $S^{*} \Omega$ (with respect to the usual cosphere bundle topology) and these functions separate points of $S^{*} \Omega$, we must have the map $m \rightarrow(x, \xi)$ continuous. In a similar manner we have the inverse mapping continuous.

Theorem 2.1 is analogous to the result for compact $\Omega$ proved by Seeley [13]. The proof is similar to that in [13], except regarding the surjectivity of the correspondence: we have used associated dual maps, ideas generalized to manifolds from Herman [8], instead of the Gohberg type estimates invoked by Seeley. In fact, it should be pointed out that the validity of Theorem 2.1 depends only on the generators of the algebra at finite points of $\Omega$ (an idea expressed more precisely in Lemma 2.2). Hence Theorem 2.1 would remain true if we were to allow generators of $\mathfrak{A}$ which do not behave asymptotically like products of vector fields in $\boldsymbol{D}$. However, to complete the analysis at hand, we must analyze the asymptotic behavior of $\boldsymbol{D}$ which we take up in the next section. 
3. The asymptotic behavior of $D$. We are interested in finding a set of generators of $\boldsymbol{D}$ at "infinite points of $\bar{\Omega}$." Let $\partial \bar{\Omega}=\bar{\Omega} \backslash \Omega$, but recall that this set only has meaning as a collection of maximal ideals for the algebra $\mathfrak{A}_{m}$. However, if $D_{1}, D_{2} \in \boldsymbol{D}$ then their pointwise inner product under the metric $g_{i j}$ defines a function $g\left(D_{1}, D_{2}\right)$ which, by the Leibniz rule for covariant derivatives, is in $A$. Thus $g\left(D_{1}, D_{2}\right)(x)$ for $x \in \partial \bar{\Omega}$ is defined.

Definition. We shall call $D_{1}, \cdots, D_{\kappa} \in \boldsymbol{D}$ an orthonormal basis for $\boldsymbol{D}$ at $x \in \partial \bar{\Omega}$ if

$$
g\left(D_{i}, D_{j}\right)(\mathbf{x})=\left\{\begin{array}{lll}
1 & \text { if } & i=j \\
0 & \text { if } & i \neq j
\end{array}\right.
$$

(ii) for every $D \in D$ thre exists constants $c_{1}, \cdots, c_{\kappa}$ such that the function $\left|D-\Sigma c_{i} D_{i}\right|^{2} \in \boldsymbol{A}$ vanishes at $x$.

Using a Gram-Schmidt type of procedure, it is not hard to verify that there exists an orthonormal basis for $D$ at each $x \in \partial \bar{\Omega}$, and that each basis contains the same number of vector fields, $\kappa=d(x)$, the "dimension" of $\boldsymbol{D}$ at $x$. Note that $\kappa \leqq n$.

We would like to be able to extend the notion of orthonormal bases to open sets in $\partial \bar{\Omega}$. In order to do so we need the following condition.

Condition $C$. The function $d: \partial \bar{\Omega} \rightarrow Z_{+}$is continuous. I.e., the dimension of $\boldsymbol{D}$ is constant on connected components of $\partial \bar{\Omega}$.

We may now prove the following.

THeOREm 3.1. Under Condition $C$, for every $x_{0} \in \partial \bar{\Omega}$ we can find an open set $U_{0} \subset \bar{\Omega}$ and real vector fields $D_{1}, \cdots, D_{\kappa} \in \boldsymbol{D}\left(\kappa=d\left(x_{0}\right)\right)$ such that

$$
\begin{gathered}
x_{0} \in U_{0} \\
g\left(D_{i}, D_{j}\right)(x)=\left\{\begin{array}{ll}
1 & \text { if } i=j \\
0 & \text { if } i \neq j
\end{array}\right\} \text { for every } x \in U_{0}
\end{gathered}
$$

(iii) for every $D \in \boldsymbol{D}$ we may find functions $a_{1}, \cdots, a_{\kappa} \in \boldsymbol{A}$ such that $D=a_{1} D_{1}+a_{2} D_{2}+\cdots+a_{k} D_{k}+D_{0}$ everywhere on $U_{0}$, where $D_{0} \in \boldsymbol{D}$ satisfies $g\left(D_{0}, D_{0}\right)(x)=0$ for all $x \in \partial \bar{\Omega} \cap U_{0}$.

Proof. Pick an orthonormal basis at $x_{0}: \widetilde{D}_{1}, \cdots, \widetilde{D}_{\kappa} \in D$. Without loss of generality we may assume $\widetilde{D}_{1}, \cdots, \widetilde{D}_{k}$ are all real vector 
fields (since otherwise we may orthogonalize the real and imaginary parts at $\left.x_{0}\right)$. Since $g\left(\widetilde{D}_{i}, \widetilde{D}_{j}\right) \in \boldsymbol{A}$ for all $i, j$, there exists an open set $U_{0} \subset \bar{\Omega}$ containing $x_{0}$ such that $\partial \bar{\Omega} \cap U_{0}$ is connected and

$$
g\left(\widetilde{D}_{i}, \widetilde{D}_{j}\right)(x)=\left\{\begin{array}{lll}
>3 / 4 & \text { if } & i=j \\
<1 / 2 \kappa & \text { if } & i \neq j
\end{array}\right.
$$

for all $x \in U_{0}$. Let $\chi \in C^{\infty}(\boldsymbol{R})$ with all derivatives bounded, $|\chi(t)| \leqq 1$ for all $t$, and

$$
\chi(t)=\left\{\begin{array}{lll}
1 & \text { if } \quad t>1 / 3 \\
0 & \text { if } \quad t<1 / 4
\end{array}\right.
$$

Let $\phi_{1}=\chi\left(g\left(\widetilde{D}_{1}, \widetilde{D}_{1}\right)\right)$. It is easy to see that $\phi_{1}\left(g\left(\widetilde{D}_{1}, \widetilde{D}_{1}\right)\right)^{-1 / 2} \in \boldsymbol{A}$ so $D_{1}=\phi_{1}\left(g\left(\widetilde{D}_{1}, \widetilde{D}_{1}\right)\right)^{-1 / 2} \widetilde{D}_{1} \in \boldsymbol{D}$ and satisfies $g\left(D_{1}, D_{1}\right)=1$ on $U_{0}$. Inductively, define $D_{j}=\phi_{j}\left(g\left(B_{j}, B_{j}\right)\right)^{-1 / 2} B_{j}$ where $\phi_{j}=\chi\left(g\left(B_{j}, B_{j}\right)\right)$ and $B_{j}=$ $\widetilde{D}_{j}-g\left(D_{1}, \widetilde{D}_{j}\right) D_{1}-\cdots-g\left(D_{j-1}, \widetilde{D}_{j}\right) D_{j-1}$. A computation shows

$$
\begin{aligned}
g\left(B_{j}, B_{j}\right) & =g\left(\widetilde{D}_{j}, \widetilde{D}_{j}\right)-g\left(D_{1}, \widetilde{D}_{j}\right)^{2}-\cdots-g\left(D_{j-1}, \widetilde{D}_{j}\right)^{2} \\
& >3 / 4-1 / 3 \kappa-1 / 3 \kappa-\cdots-1 / 3 \kappa>1 / 3
\end{aligned}
$$

for $x \in U_{0}$, so $D_{j} \in \boldsymbol{D}$. Hence $D_{1}, \cdots, D_{\kappa}$ is orthogonal at every $x \in U_{0}$, i.e., we have (ii). Now let $a_{j}=g\left(D, D_{j}\right) \in A$, and let $D_{0}=D-$ $\sum_{j=1}^{\kappa} a_{j} D_{j}$. Then

$$
g\left(D_{0}, D_{j}\right)=g\left(D-\Sigma a_{i} D_{i}, D_{j}\right)=a_{j}-a_{j}=0
$$

implies $D_{0}$ is never a linear combination of $D_{1}, \cdots, D_{\kappa}$ on $U_{0}$. Hence by Condition $\mathrm{C}$ and the connectedness of $\partial \bar{\Omega} \cap U_{0}$, we must have $g\left(D_{0}, D_{0}\right)(x)=0$ for all $x \in \partial \bar{\Omega} \cap U_{0}$.

4. The formal algebra symbol and maximal ideals at infinity. For $L=\sum_{\alpha=0}^{N} l_{\alpha} \nabla^{\alpha} \in \boldsymbol{T}^{N}$, let us define the formal symbol

$$
\widetilde{\sigma}_{L \Lambda^{N}}(x, \xi)=\left(\sum_{\alpha=0}^{N} l_{\alpha}(x) \xi^{\alpha}\right)\left(1+|\xi|^{2}\right)^{-N / 2}=\widetilde{\sigma}_{A^{N} L}(x, \xi)
$$

which is a continuous function on the cotangent bundle $T^{*} \Omega$. (Recall from $\S 2$ that the notation $l_{\alpha} \xi^{\alpha}$ means the function $l_{\alpha}^{i_{1} \cdots i_{\alpha}}(x) \xi_{i_{1}} \cdots \xi_{i_{\alpha}}$.) Note that the boundedness of the coefficient tensors of $L$ implies $\widetilde{\sigma}_{L N}$ is bounded on $T^{*} \Omega$. The formal symbols for operators $L \Lambda^{N}$ and $\Lambda^{N} L$ in $\mathfrak{Q}^{0}$ generate a $C^{*}$-subalgebra $\mathscr{F}$ of the algebra of bounded continuous functions on $T^{*} \Omega$. Hence $\mathscr{F}$ is isometrically *-isomorphic to $C\left(P^{*} \Omega\right)$, where $P^{*} \Omega$ denotes some compactification of $T^{*} \Omega$. The injection $\mathfrak{A}_{m} \rightarrow \mathscr{F}$ yields a continuous surjection $\pi$ : $P^{*} \Omega \rightarrow \bar{\Omega}$. Unfortunately the map $\widetilde{\sigma}: \mathfrak{Q}^{0} \rightarrow C\left(P^{*} \Omega\right)$ is not an algebra homomorphism: for example $\widetilde{\sigma}_{A} \widetilde{\sigma}_{D_{1} D_{2} A^{2}}=\left(b^{i} c^{j} \xi_{i} \xi_{j}+b^{i} c^{j},{ }_{i} \hat{\xi}_{j}\right)\left(1+|\xi|^{2}\right)^{-3 / 2}$ 
but $\tilde{\sigma}_{A D_{1}} \tilde{\sigma}_{D_{2} \Lambda^{2}}=\left(\boldsymbol{b}^{i} c^{j} \xi_{i} \xi_{j}\right)\left(1+|\xi|^{2}\right)^{-3 / 2} \quad$ where $\quad D_{1}=b^{i} V_{i}, \quad D_{2}=c^{j} \nabla_{j} \in \boldsymbol{D}$. However, if we restric our functions to the subset $\partial P^{*} \Omega=P^{*} \Omega \backslash T^{*} \Omega$, we obtain an algebra homomorphism $\dot{\sigma}: \mathfrak{A}^{0} \rightarrow C\left(\partial P^{*} \Omega\right)$ and we call $\dot{\sigma}_{A}=\left.\tilde{\sigma}_{A}\right|_{\partial P * \Omega}$ the formal algebra symbol of $A \in \mathfrak{X}^{0}$.

It is evident that the cosphere bundle $S^{*} \Omega$ is homeomorphic to $\pi^{-1}(\Omega)$ by the map $(x, \xi) \rightarrow \lim _{r \rightarrow \infty}(x, r \xi) \in \partial P^{*} \Omega$. Theorem 2.1 may be interpreted as providing a continuous injection $\theta: S \rightarrow \partial P^{*} \Omega$ such that

$$
\dot{\sigma}_{A}(\theta(m))=\sigma_{A}(m)
$$

for $m \in S$ and operators $A \in \mathfrak{U}^{0}$. The main result of this paper extends this results as follows.

THEOREM 4.1. Under Condition C, there exists continuous injection $\theta: \boldsymbol{M} \rightarrow \partial P^{*} \Omega$ such that

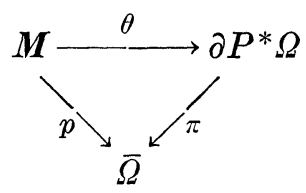

is commutative, and (4.2) holds for all $m \in M$ and $A \in \mathfrak{H}^{0}$. Thus a sufficient condition that $L: \mathfrak{S}_{N} \rightarrow \mathfrak{S}$ be Fredholm is that $\dot{\sigma}_{L \Lambda^{N}} \neq 0$ on $\partial P^{*} \Omega$.

Before extending $\theta$ to $\boldsymbol{M}_{\infty}=\boldsymbol{M} \backslash \boldsymbol{S}$, we first must analyze $C(\boldsymbol{M})$ a little more closely. Let $\boldsymbol{M}_{p}$ denote the closure in $\boldsymbol{M}$ of $\boldsymbol{S}$ and $\boldsymbol{M}_{s}=\boldsymbol{M} \backslash \boldsymbol{M}_{p}$. We may now prove the following.

LeMma 4.2. $\boldsymbol{M}_{p}=\left\{m \in \boldsymbol{M}: \sigma_{\Lambda}(m)=0\right\}$.

Proof. By Theorem 2.1 it is clear that $M_{p} \subset\left\{m \in M: \sigma_{\Lambda}(m)=0\right\}$. Let $\mathscr{J}=\left\{A \in \mathfrak{A}: \sigma_{A}=0\right.$ on $\left.\boldsymbol{M}_{p}\right\}$. Then $\mathscr{J} / \mathscr{K}$ is a $C^{*}$-algebra (without unit) which is isomorphic to the algebra of continuous functions on $M_{s}$ which vanish at infinity. Since $\Lambda$ is the only generator of $\mathfrak{Y}^{0}$ in $\mathscr{J}, \mathscr{J}$ must be generated by operators of the form $A \Lambda$ and $\Lambda A$ where $A \in \mathfrak{U}^{0}$. So if $\sigma_{A}(m)=0$ for $m \in \boldsymbol{M}_{s}$, then $\sigma_{B}(m)=0$ for all $B \in \mathscr{J}$ which is impossible.

LEMMA 4.3. If $L \in \boldsymbol{T}_{0}^{N}$, then $\sigma_{L A^{N}}=0$ on $\boldsymbol{M}_{\infty}$.

Proof. If $m \in \boldsymbol{M}_{\infty} \cap \boldsymbol{M}_{p}$, let $m_{\beta}$ be a net in $\boldsymbol{S}$ converging to $m$. Then using Theorem 2.1 we conclude that $\sigma_{L \Lambda^{N}}\left(m_{\beta}\right) \rightarrow 0=\sigma_{L \Lambda^{N}}(m)$. 
If $m \in M_{s}$, then $\sigma_{\Lambda}(m)=0$ by Lemma 4.2. But $\sigma_{L A^{N}}(m) \sigma_{\Lambda}(m)=\sigma_{L \Lambda^{N+1}}$ $(m)=0$ since $L A^{N+1} \in \mathscr{K}$; so $\sigma_{L A^{N}}(m)=0$.

Now let $m_{0} \in \boldsymbol{M}_{\infty}$ with $x_{0}=p\left(m_{0}\right) \in \partial \bar{\Omega}$. By the methods used in the proof of Theorem 3.1, it is possible to construct (in addition to $U_{0}$ ) a closed neighborhood $U \subset U_{0}$ of $x_{0}$, and $\phi \in \mathfrak{A}_{m}$ satisfying $\phi=1$ on $U, 0 \leqq \phi \leqq 1$ on $\Omega$, and $\operatorname{supp} \phi \subset U_{0}$. Let $\mathfrak{A}_{\phi}, p_{\phi}, M_{\phi}, N_{\phi}$, and $N$ all be as described in Lemma 2.2. Furthermore, let $U_{\infty}=\partial \bar{\Omega} \cap U$ and $N_{\infty}=p^{-1}\left(U_{\infty}\right)$. By Lemma 2.2 we will also consider $N_{\infty} \subset M_{\phi}$. Let $D_{1}, \cdots, D_{\kappa} \in D\left(\kappa=d\left(x_{0}\right)\right)$ be real vector fields as in Theorem 3.1, with expressions in local coordinates $D_{\nu}=b_{\nu}^{j}\left(\partial / \partial x^{j}\right)$ for $\nu=1, \cdots, \kappa$. Let $S_{\nu}=-i \phi D_{\nu} \Lambda \in \mathfrak{A}_{\phi}$ for $\nu=1, \cdots, \kappa$. We define a contravariant tensor $h^{j k}$ by

$$
g^{j k}=\sum_{\nu=1}^{\kappa} b_{\nu}^{j} b_{\nu}^{k}+h^{j k} .
$$

If $(x, \xi)$ is a complex covector with $x \in U_{0}$ and $|\xi|^{2}=1$, then

$$
\sum_{\nu=1}^{\kappa} b_{\nu}^{j} \xi_{j} b_{\nu}^{k} \bar{\xi}_{k} \leqq 1
$$

since the $D_{\nu}$ form an orthonormal set on $U_{0}$. Hence $h^{j k} \xi_{j} \bar{\xi}_{k} \geqq 0$, i.e., $h^{j k}$ is positive semidefinite. We shall assume henceforth that $h^{j k}$ is not identically zero, i.e., that $\kappa<n$. (See Remark 4.6 below for the case $\kappa=n$.) From (4.4) we obtain

$$
\nabla_{j} \phi^{2} g^{j k} \nabla_{k}=\sum_{\nu=1}^{k} \nabla_{j} \phi^{2} b_{\nu}^{j} b_{\nu}^{k} \nabla_{k}+\nabla_{j} \phi^{2} h^{j k} \nabla_{k} .
$$

Since

$$
\begin{aligned}
\Lambda \nabla_{j} \phi^{2} g^{j k} \nabla_{k} \Lambda & \equiv \Lambda \phi^{2} \Delta \Lambda(\bmod \mathscr{K}) \\
& \equiv \phi^{2} \Lambda \Delta \Lambda(\bmod \mathscr{K}) \\
& =\phi^{2} \Lambda^{2}-\phi^{2}
\end{aligned}
$$

and

$$
\begin{aligned}
\Lambda\left(\nabla_{j} \phi^{2} b_{\nu}^{j}\right)\left(b_{\nu}^{k} \nabla_{k}\right) \Lambda & =-\Lambda\left(\nabla_{j} i \phi b_{\nu}^{j}\right)\left(i \phi b_{\nu}^{k} \nabla_{k}\right) \Lambda \\
& =-S_{\nu}^{*} S_{\nu} \\
& =-S_{\nu}^{2}(\bmod \mathscr{C})
\end{aligned}
$$

we get after multiplying (4.5) on the left and right by $A$ and rearranging:

$$
\phi^{2} \equiv \phi^{2} \Lambda^{2}-\Lambda H \Lambda+\sum_{\nu=1}^{\kappa} S_{\nu}^{2}(\bmod \mathscr{K})
$$

where $H$ denotes the operator $\nabla_{j} \phi^{2} h^{j k} \nabla_{k}$. The positive semi-definite- 
ness of the tensor $h^{j k}$ implies

$$
\langle\Lambda H \Lambda u, u\rangle=-\int_{\Omega} \phi^{2} h^{j k}\left(\nabla_{k} \Lambda u\right)\left(\overline{\left.\nabla_{j} \Lambda u\right)} d \mu \leqq 0\right.
$$

for $u \in C_{0}^{\infty}(\Omega)$, so the operator $\Lambda H \Lambda$ is nonpositive. Hence $\phi^{2} \Lambda^{2}-\Lambda H \Lambda$ is nonnegative and we may define $S_{0}=\left(\phi^{2} \Lambda^{2}-\Lambda H \Lambda\right)^{-1 / 2}$. From (4.8) we conclude $\Lambda H \Lambda, S_{0} \in \mathfrak{A}_{\phi}$ and

$$
\phi^{2} \equiv \sum_{\nu=0}^{\kappa} S_{\nu}^{2}(\bmod \mathscr{K}) \text {. }
$$

If we let $S_{+}^{\kappa}=\left\{\zeta=\left(\zeta_{0}, \cdots, \zeta_{\kappa}\right) \in R^{\kappa+1}: \sum_{\nu=0}^{\kappa} \zeta_{\nu}^{2}=1\right.$ and $\left.\zeta_{0} \geqq 0\right\}$, then we are now able to prove the following.

Proposition 4.4. There exists a continuous injection

$$
\omega_{1}: N_{\infty} \longrightarrow U_{\infty} \times[0,1] \times S_{+}^{\kappa}
$$

such that $\omega_{1}(n)=(x, r, \zeta)$ satisfies (i) $\sigma_{a}(n)=a(x)$, (ii) $\sigma_{\Lambda}(n)=r$, (iii) $\sigma_{S_{\nu}}(n)=\zeta_{\nu}(\nu=0,1, \cdots, \kappa)$, and (iv) $\zeta_{0} \geqq r$.

Proof. Since $N_{\infty}$ is a closed subset of $M_{\phi}, \mathscr{J}_{\phi}=\left\{A \in \mathfrak{A}_{\phi}: \sigma_{A}^{\phi}=0\right.$ on $\left.N_{\infty}\right\}$ is a closed ideal of $\mathfrak{U}_{\phi}$, and $\mathfrak{A}_{\phi} / \mathscr{J}_{\phi} \simeq C\left(N_{\infty}\right)$. Furthermore, using $N_{\infty} \subset M_{\infty}$ and Lemma $4.3, \mathfrak{A}_{\phi} / \mathscr{J}_{\phi}$ is generated by $a+\mathscr{J}_{\phi}(a \in A)$, $\phi \Lambda+\mathscr{J}_{\phi}$, and $S_{\nu}+\mathscr{J}_{\phi}(\nu=1, \cdots, \kappa)$. Thus if we let $\mathscr{B}_{m}$ be the $C^{*}$-algebra generated by $\mathfrak{A}_{m}$ and $\mathscr{J}_{\phi}$, then $\mathscr{B}_{m} / \mathscr{J}_{\phi}$ is a commutative $C^{*}$-algebra isometrically *-isomorphic to $C\left(U_{\infty}\right)$. Also if we let $\mathfrak{A}_{\phi}^{\#}$ be the $C^{*}$-algebra generated by $\phi \Lambda, S_{\nu}(\nu=0,1, \cdots, \kappa)$, and $\mathscr{J}_{\phi}$; then $\mathfrak{X}_{\phi}^{*} / \mathscr{J}_{\phi}$ is a commutative $C^{*}$-algebra (with unit using (4.8) and the fact that $\left.\phi \equiv 1\left(\bmod \mathscr{J}_{\phi}\right)\right)$. In fact, since $\mathfrak{2}_{\phi}^{*} / \mathscr{J}_{\phi}$ is finitely generated, it has maximal ideal space $N_{\infty}^{\sharp}$ homeomorphic to the joint spectrum of its generators (c.f. [12]). If we let $\sigma^{\sharp}: \mathscr{Y}_{\phi}^{\sharp} \rightarrow C\left(N_{\infty}^{\sharp}\right)$ denote the symbol homomorphism, then (4.9) implies $\sigma_{A H A}^{\#} \leqq 0$, and so by (4.8), $0 \leqq \sigma_{\phi^{2} A^{2}}^{\#} \leqq 1$. By (4.10) we also have $\sum_{y=0}^{k}\left(\sigma_{S_{\nu}}^{\#}\right)^{2}=1$. Thus there is an injection

$$
N_{\infty}^{\sharp} \longrightarrow[0,1] \times S_{+}^{\kappa}
$$

where $n \rightarrow(r, \zeta)$ satisfies $\sigma_{\phi 1}^{\sharp}(n)=r$ and $\sigma_{S_{\nu}}^{*}(n)=\zeta_{\nu}$. Since $\mathfrak{A}_{\phi} / \mathscr{J}_{\phi}$ is generated by $\mathscr{B}_{n} / \mathscr{J}_{\phi}$ and $\mathfrak{U}_{\phi}^{\#} / \mathscr{J}_{\phi}$, we can use Herman's Lemma (c.f. [8], Theorem 1) to conclude the existence of a continuous injection $N_{\infty} \rightarrow U_{\infty} \times N_{\infty}^{\#}$ and composition with (4.12) yields a continuous injection $\omega_{\phi}: N_{\infty} \rightarrow U_{\infty} \times[0,1] \times S_{+}^{\kappa}$ satisfying $\sigma_{a}^{\phi}(n)=a(x), \sigma_{\phi \Lambda}^{\phi}(n)=r$, and $\sigma(n)_{\nu}^{\phi}=\zeta_{\nu}$. When we view $\boldsymbol{N}_{\infty} \subset \boldsymbol{M}$ (i.e., compose $\omega_{\phi}$ with the homeomorphism $p^{-1}(U) \simeq p_{\phi}^{-1}(U)$ ), we obtain the map $\omega_{\dot{\phi}}$ of (4.11) satisfying (i)-(iii). Condition (iv) is simply a consequence of $\Lambda^{2} \leqq$ $\Lambda^{2}-\Lambda H \Lambda$. 
In a similar manner we can let $P^{*} U=\pi^{-1}(U)$ and $P^{*} U_{\infty}=$ $P^{*} U \cap P^{*} \Omega_{\infty}=\pi^{-1}\left(U_{\infty}\right)$. Applying the above argument to the function algebra $\mathscr{F}_{\phi}$ generated by the formal symbolsof the generators of $\mathfrak{A}_{\phi}$ yields

Proposition 4.5. There exists a continuous injection

$$
\omega_{2}: P^{*} U_{\infty} \longrightarrow U_{\infty} \times[0,1] \times S_{*}^{\wedge}
$$

such that for $\omega_{2}(p)=(x, r, \zeta)$ we have (i) $\dot{\sigma}_{a}(p)=a(x)$, (ii) $\dot{\sigma}_{A}(p)=r$, (iii) $\dot{\sigma}_{S_{\nu}}(p)=\zeta_{\nu}(\nu=0,1, \cdots, \kappa)$, and (iv) $\zeta_{0} \geqq r$. In fact, every $(x, r, \zeta)$ in $U_{\infty} \times[0,1] \times S_{+}^{\kappa}$ satisfying (iv) is in the range of $\omega_{2}$.

Proof. To prove the last statement, let $x_{\beta} \in \Omega$ denote a net converging to $x$. Let $\xi_{\beta} \in \pi^{-1}\left(x_{\beta}\right)$ satisfy

$$
b_{\nu}^{j}\left(\xi_{\beta}\right)_{j} \vartheta=\zeta_{\nu} \quad(\nu=1, \cdots, \kappa) .
$$

But this implies by (4.4) that

$$
\left\{\left|\xi_{\beta}\right|^{2}-h^{i j}\left(\xi_{\beta}\right)_{i}\left(\xi_{\beta}\right)_{j}\right\} r^{2}=\sum_{\nu=1}^{\kappa} \zeta_{\nu}^{2}
$$

or

$$
\left|\xi_{\beta}\right|^{2}=\frac{1}{r^{2}}-\frac{\left(\zeta_{0}\right)^{2}}{r^{2}}+h^{i j}\left(\xi_{\beta}\right)_{i}\left(\xi_{\beta}\right)_{j} .
$$

The last term in (4.15) is nonnegative, so by adding something to $\xi_{\beta}$ from the kernel of the system (4.14), which is nontrivial since we are assuming $\kappa<n$, we can also require

$$
\left|\xi_{\beta}\right|^{2}=\frac{1}{r^{2}}-1
$$

provided $\zeta_{0} \geqq r$. (Note: if $r=0$, take $\xi_{\beta}=\lim _{r \rightarrow \infty} r \xi_{\beta}^{\prime} \in \pi^{-1}\left(x_{\beta}\right)$ where $\xi_{\beta}^{\prime}$ solves $b_{\nu}^{j}\left(\xi_{\beta}^{\prime}\right)_{j}=\zeta_{\nu}$.) Observe that for each $\beta, \widetilde{\sigma}_{A}\left(x_{\beta}, \xi_{\beta}\right)=r$ and $\tilde{\sigma}_{S_{\nu}}\left(x_{\beta}, \xi_{\beta}\right)=\zeta_{\nu}(\nu=1, \cdots, \kappa)$; hence also $\left[\widetilde{\sigma}_{S_{0}}\left(x_{\beta}, \xi_{\beta}\right)\right]^{2}=1-\sum_{\nu=1}^{k} \zeta_{\nu}^{2}=\zeta_{0}^{2}$ for every $\beta$. Thus $\left(x_{\beta}, \xi_{\beta}\right)$ must converge to a point $p \in P^{*} \Omega_{\infty}$ satisfying $\omega_{2}(p)=(x, r, \zeta)$.

These propositions easily imply our main result.

Proof of Theorem 4.1. Note that $\omega_{2}^{-1} \circ \omega_{1}$ provides a continuous injection $\theta: N_{\infty} \rightarrow P^{*} \Omega_{\infty}$. It is clear that the value $\theta(n) \in \partial P^{*} \Omega$ does not depend on the choice of neighborhood $U$, so we obtain a continuous injection $\theta: \boldsymbol{M}_{\infty} \rightarrow \partial P^{*} \Omega$. Letting $m \in \boldsymbol{M}_{\infty} \cap \boldsymbol{M}_{p}$ and $m_{\beta} \in \boldsymbol{S}$ denote a net converging to $m$, it is easy to check that $\dot{\sigma}_{A}(\theta(m))=$ 
$\lim _{\beta} \dot{\sigma}_{A}\left(\theta\left(m_{\beta}\right)\right)$ for every $A \in \mathfrak{A}^{0}$, so we obtain a continuous injection $\theta: M \rightarrow \partial P^{*} \Omega$ satisfying (4.2).

REMARK 4.6. It was assumed above that $\kappa<n$. Actually, the case $\kappa=n$ is easier, for then we obtain from

$$
g^{j k}=\sum_{\nu=1}^{n} b_{\nu}^{j} b_{\nu}^{k}
$$

that

$$
\begin{aligned}
\phi^{2} & \equiv \phi^{2} \Lambda^{2}+\sum_{\nu=1}^{n} S_{\nu}^{2}(\bmod \mathscr{K}) \\
& \equiv \sum_{\nu=0}^{n} S_{\nu}^{2}(\bmod \mathscr{K})
\end{aligned}
$$

after defining $S_{0}=\phi \Lambda$. In place of Proposition 4.4, the same proof establishes a continuous injection

$$
\omega_{1}: N_{\infty} \longrightarrow U_{\infty} \times S_{+}^{n}
$$

satisfying $\omega_{1}(n)=(x, \zeta)$ with $\sigma_{a}(n)=a(x)$ and $\sigma_{S_{\nu}}(n)=\zeta_{\nu}(\nu=0, \cdots, n)$. Similarly we get a continuous injection

$$
\omega_{2}: P^{*} U_{\infty} \longrightarrow U_{\infty} \times S_{+}^{n}
$$

satisfying $\omega_{2}(n)=(x, \zeta)$ with $\dot{\sigma}_{a}(n)=a(x)$ and $\dot{\sigma}_{S_{\nu}}(n)=\zeta_{\nu}(\nu=0, \cdots, n)$. In fact, imitating the proof of Proposition 4.5 establishes that $\left(4.13^{\prime}\right)$ is surjective, and the proof of Theorem 4.1 follows as above.

\section{REFERENCES}

1. T. Aubin, Espaces de Sobolev sur les varietes riemanniennes, preprint.

2. M. Cantor, Spaces of functions with assymptotic conditions on $R^{n}$, Indiana Univ. Math. J., 24, No. 9, (1975), 897-902.

3. H. O. Cordes, Self-adjointness of powers of elliptic operators on noncompact manifolds, Math. Ann., 195 (1972), 257-272.

4. H. O. Cordes and E. Herman, Gel'fand theory of pseudo-differential operators, Amer.

J. Math., 90 (1968), 681-717.

5. H. O. Cordes and R. C. McOwen, The $C^{*}$-algebra of a singular elliptic problem on a noncompact riemannian manifold, Math. Zeit., 153 (1977), 101-116.

6. - Remarks on singular elliptic theory for complete riemannian manifolds, 70, No. 1, (1977), 133-141.

7. L. P. Eisenhart, Riemannian Geometry, Princeton University Press, 1926.

8. E. Herman, The symbol of the algebra of singular integral operators, J. Math. Mech., 15 (1966), 147-156.

9. R. Jackson, Canonical operators and lower-order symbols, Amer. Math. Soc. Memoirs, (1973).

10. S. Kobayashi and K. Nomizu, Foundations of Differential Geometry, Vol. I, NewYork-London, Interscience, 1963.

11. R. C. McOwen, The behavior of the laplacian on weightad Sobolev spaces, Comm. Pure and App. Math., (to appear). 
12. C. E. Rickart, Banach Algebras, Princeton, Van Nostrand, 1960.

13. R. T. Seeley, Integro-differential operators on vector bundles, Trans. Amer. Math. Soc., 117 (1965), 167-204.

Received April 11, 1979.

NeW YoRK UNIVERSITY

CoURANT INSTITUTE

NEW YoRK, NY 10012 



\section{PACIFIC JOURNAL OF MATHEMATICS}

\section{EDITORS}

DONALD BABBITT (Managing Editor)

University of Galifornia

Los Angeles, California 90024

HUGo RossI

University of Utah

Salt Lake City, UT 84112

C. C. MOORE AND ANDREW OGG

University of California

Berkeley, CA 94720

\section{J. DUGUNDJI}

Department of Mathematics University of Southern California Los Angeles, California 90007

R. FINN AND J. MILGRAM Stanford University Stanford, California 94305

ASSOCIATE EDITORS
E. F. BECKENBACH
B. H. NeumanN
F. WoLF
K. YosHIDA

\section{SUPPORTING INSTITUTIONS}

UNIVERSITY OF BRITISH COLUMBIA UNIVERSITY OF SOUTHERN CALIFONIA CALIFORNIA INSTITUTE OF TECHNOLOGY UNIVERSITY OF CALIFORNIA MONTANA STATE UNIVERSITY STANFORD UNIVERSITY UNIVERSITY OF HAWAII UNIVERSITY OF TOKYO UNIVERSITY OF NEVADA, RENO UNIVERSITY OF UTAH NEW MEXICO STATE UNIVERSITY WASHINGTON STATE UNIVERSITY OREGON STATE UNIVERSITY UNIVERSITY OF OREGON UNIVERSITY OF WASHINGTON 


\section{Pacific Journal of Mathematics \\ Vol. 87, No. $1 \quad$ January, 1980}

Spiros Argyros, A decomposition of complete Boolean algebras ..........

Gerald A. Beer, The approximation of upper semicontinuous multifunctions

by step multifunctions . . . . ....................

Ehrhard Behrends and Richard Evans, Multiplicity theory for Boolean

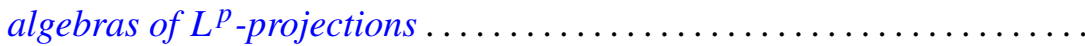

Man-Duen Choi, The full $C^{*}$-algebra of the free group on two

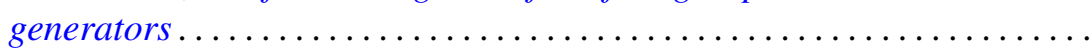

Jen-Chung Chuan, Axioms for closed left ideals in a $C^{*}$-algebra . . . . . . . .

Jo-Ann Deborah Cohen, The strong approximation theorem and locally

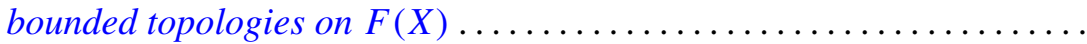

Eugene Harrison Gover and Mark Bernard Ramras, Increasing sequences of Betti numbers............................

Morton Edward Harris, Finite groups having an involution centralizer with

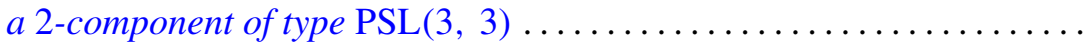

Valéria Botelho de Magalhães Iório, Hopf $C^{*}$-algebras and locally compact

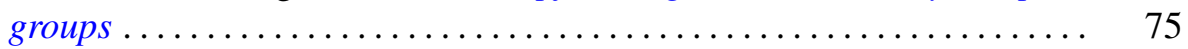

Roy Andrew Johnson, Nearly Borel sets and product measures . . . . . . . . . .

Lowell Edwin Jones, Construction of $Z_{p}$-actions on manifolds . . . . . . . . .

Manuel Lerman and Robert Irving Soare, $d$-simple sets, small sets, and

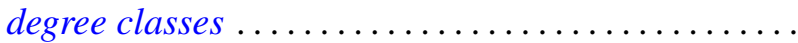

Philip W. McCartney, Neighborly bushes and the Radon-Nikodým property

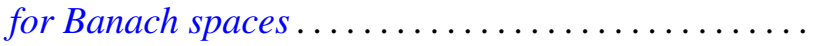

Robert Colman McOwen, Fredholm theory of partial differential equations on complete Riemannian manifolds.

Ernest A. Michael and Carl Preston Pixley, A unified theorem on continuous selections.

Ernest A. Michael, Continuous selections and finite-dimensional sets .

Vassili Nestoridis, Inner functions: noninvariant connected components...

Bun Wong, A maximum principle on Clifford torus and nonexistence of proper holomorphic map from the ball to polydisc.

Steve Wright, Similarity orbits of approximately finite $C^{*}$-algebras . . .

Kenjiro Yanagi, On some fixed point theorems for multivalued

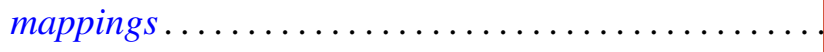

Wieslaw Zelazko, A characterization of LC-nonremovable ideals in commutative Banach algebras 